\title{
Empirical Validation and Psychometric Evaluation of the Brief Fear of Negative Evaluation Scale in Patients With Social Anxiety Disorder
}

\author{
Justin W. Weeks and Richard G. Heimberg \\ Temple University \\ David M. Fresco \\ Kent State University \\ Trevor A. Hart \\ Cynthia L. Turk \\ York University \\ LaSalle University
}

Franklin R. Schneier and Michael R. Liebowitz

New York State Psychiatric Institute and Columbia University

\begin{abstract}
The Brief Fear of Negative Evaluation Scale (BFNE; M. R. Leary, 1983a) is often used to assess fear of negative evaluation, the core feature of social anxiety disorder. However, few studies have examined its psychometric properties in large samples of socially anxious patients. Although the BFNE yields a single total score, confirmatory factor analysis indicated a 2-factor solution to be more appropriate, with the 1st factor consisting of all straightforwardly worded items (BFNE-S) and the 2nd of all reverse-scored items (BFNE-R). Support was obtained for the convergent and discriminant validity of the BFNE and BFNE-S, but not the BFNE-R. These results suggest that standard scoring of the BFNE may not be optimal for patients with social anxiety disorder.
\end{abstract}

Keywords: social anxiety disorder, social phobia, anxiety disorders, fear of negative evaluation, psychometric characteristics

A key proposition of cognitive-behavioral models of social anxiety (Clark \& Wells, 1995; Rapee \& Heimberg, 1997) is that social anxiety is, in part, a response to perceived negative evaluation by others. The construct of fear of negative evaluation consists of feelings of apprehension about others' evaluations, distress over these negative evaluations, and the expectation that others will evaluate one negatively (Watson \& Friend, 1969). This construct is distinct from, but closely related to, social anxiety. Specifically, fear of negative evaluation pertains to the sense of dread associated with being evaluated unfavorably while antici-

Justin W. Weeks and Richard G. Heimberg, Adult Anxiety Clinic of Temple, Department of Psychology, Temple University; David M. Fresco, Department of Psychology, Kent State University; Trevor A. Hart, Department of Psychology, York University, Toronto, Ontario, Canada; Cynthia L. Turk, Department of Psychology, LaSalle University; Franklin R. Schneier and Michael R. Liebowitz, Anxiety Disorders Clinic, New York State Psychiatric Institute, New York, and Department of Psychiatry, Columbia University.

Portions of this article were presented at the annual meeting of the Association for the Advancement of Behavior Therapy, Reno, Nevada, in November 2002, and the Anxiety Disorders Association of America, Toronto, Ontario, Canada, in March 2003.

We thank Thomas L. Rodebaugh III, Lauren B. Alloy, and Robert Fauber for comments on an earlier version of this article.

Correspondence concerning this article should be addressed to Richard G. Heimberg, Department of Psychology, Temple University, Weiss Hall, 1701 North 13th Street, Philadelphia, PA 19122. E-mail: heimberg@temple.edu pating or participating in a social situation, whereas social anxiety pertains to affective reactions to these situations.

Empirical support for the proposition that social anxiety is a response to fear of negative evaluation has been impressive. For example, socially anxious individuals exhibit a variety of subtle behaviors aimed at avoiding potential negative evaluation (Wells et al., 1995). Socially anxious individuals have also consistently shown preferential allocation of attentional resources to the detection of social-evaluative threat. This phenomenon has been demonstrated by using a number of cognitive-experimental approaches for the assessment of attentional processes, including the dot-probe paradigm (e.g., Asmundson \& Stein, 1994) and the modified Stroop task (e.g., Hope, Rapee, Heimberg, \& Dombeck, 1990; see Heinrichs \& Hofmann, 2001, for a review). In addition, socially anxious persons rate their own behavior during impromptu speeches and social interactions more poorly than do observers (Lundh \& Sperling, 2002; Rapee \& Lim, 1992; Stopa \& Clark, 1993), and fear of negative evaluation is associated with this rating discrepancy (Rapee \& Lim, 1992; Rodebaugh \& Chambless, 2002). It has been suggested that this phenomenon reflects a basic perceptual and/or processing difference between socially anxious and nonanxious individuals, which occurs specifically for the evaluation of social performance (Heinrichs \& Hofmann, 2001). Furthermore, recent evidence suggests that fear of negative evaluation is a partially heritable trait related to other dimensions that may also be related to social anxiety, such as trait anxiousness, submissiveness, and social avoidance (Stein, Jang, \& Livesley, 2002). 
The Fear of Negative Evaluation Scale (FNE) is a self-report questionnaire that was designed to assess this construct (Watson \& Friend, 1969) and that has been widely used for this purpose. The FNE has been shown to be highly reliable, and FNE scores predict numerous aspects of socially anxious behavior (Friend \& Gilbert, 1973; Smith \& Sarason, 1975; Watson \& Friend, 1969). For example, high scorers on the FNE are more likely to catastrophize in reaction to mildly negative social events (Stopa \& Clark, 2001), to identify others' facial expressions as negative (Winton, Clark, \& Edelmann, 1995), to overestimate the observability of negative social characteristics (e.g., awkwardness, long gaps in speech) and underestimate the observability of positive social characteristics (e.g., confidence, self-assurance) they exhibit during public speaking (Mansell \& Clark, 1999), and to focus attention on social threat words (Stopa \& Clark, 2001). Notwithstanding its relevance to the study of social anxiety, several potential limitations to the usefulness of the FNE exist. Some researchers (e.g., Leary, 1983a) have argued that its length (30 items) may tax respondents' patience and endurance. Although the total number of items included in the FNE is modest in comparison to other psychological assessments, its highly redundant item content makes it a very inefficient assessment device.

Concerns have also been raised regarding the treatment sensitivity of the FNE (Leary, 1983a; Saluck et al., 2000). Because of its true-false format, individuals without clinically significant fear of negative evaluation or who have improved significantly in response to treatment for social anxiety could respond positively to FNE questions intended to reflect problematic fear of negative evaluation (e.g., it would not be unreasonable for a person with little social anxiety to respond "true" to the item "Sometimes I think I am too concerned with what other people think of me"; Watson \& Friend, 1969). These individuals might give a less extreme response if provided with multiple response options. Furthermore, Heimberg (1994) has previously argued that the FNE confounds the assessment of cognition and anxiety. To illustrate, the FNE contains the item "I become tense and jittery if I know someone is sizing me up" (Watson \& Friend, 1969), which clearly pertains to bodily manifestations of anxiety as well as the cognitive content that is the proper domain of the FNE. These concerns were addressed in part by Leary (1983a), who developed a brief version of the FNE (BFNE).

The 12-item BFNE uses a 5-point Likert-type rating scale, ranging from 1 (not at all characteristic of me) to 5 (extremely characteristic of $m e$ ), rather than the true-false format of the original FNE. Undergraduates' responses were correlated highly with the original scale $(r=.96)$, and the BFNE demonstrated both high internal consistency $(\alpha=.90-.91)$ and 4-week test-retest reliability $(r=.75)$ in undergraduate samples (Leary, 1983a; Miller, 1995). Undergraduate BFNE scores also correlated with two measures of social anxiety, the Social Avoidance and Distress Scale (Watson \& Friend, 1969) and the Interaction Anxiousness Scale (Leary, 1983b), providing some preliminary evidence of convergent validity. However, the item content of the BFNE appears to more clearly reflect fear of negative evaluation than social anxiety, and Miller (1995) has provided empirical support for this assertion. Specifically, in an undergraduate sample, factor analysis indicated that the BFNE and measures of social anxiety loaded on distinct (but correlated) factors.
A recent study by Rodebaugh et al. (2004) provided additional information about the psychometric properties of the BFNE in an undergraduate sample. The BFNE was more sensitive to differing degrees of fear of negative evaluation than was the FNE in an item-response theory analysis. Also, confirmatory factor analysis of the BFNE demonstrated that a two-factor structure, with one factor consisting of all straightforwardly worded items and the second factor consisting of all reverse-scored items, provided the best fit to the data. However, Rodebaugh et al. (2004) proposed that this factor structure may not be a reflection of two distinct, underlying constructs but rather an artifact of the wording of the questions. Specifically, the two factors may represent a single construct assessed by two sets of items that use different methods (i.e., straightforward and reverse scored).

Despite these favorable preliminary data on the BFNE, it is necessary to evaluate its psychometric characteristics for all populations for which it may be used. It is noteworthy, therefore, that only one small study $(N=18)$ has examined the psychometric properties of the BFNE in patients with social anxiety disorder (Saluck et al., 2000). Saluck et al. (2000) reported that the BFNE correlated highly with the original FNE $(r=.91)$ and was moderately correlated with several measures of social anxiety and avoidance. Although a strong correlation between the BFNE and the Beck Depression Inventory (BDI; $r=.61$ ) suggested that the BFNE may not adequately distinguish between depressed mood and social anxiety, this relationship must be viewed with caution given the small sample size. ${ }^{1}$

A study using both a larger sample of patients with social anxiety disorder and a nonanxious comparison group is an essential next step in establishing the reliability, validity, and clinical utility of the BFNE. To this end, the present study evaluated the BFNE responses of 165 participants presenting for treatment of social anxiety disorder, as well as the posttreatment responses of 54 patients who completed a course of cognitive-behavioral group therapy (CBGT). The BFNE responses of 32 nonanxious control participants were also evaluated. The following questions were addressed: (a) What is the factor structure of the BFNE in patients with social anxiety disorder? Is it best described by the two-factor model derived from an undergraduate sample by Rodebaugh et al. (2004)? (b) What are the characteristics of the distributions of the BFNE total score and scores on subscales based on the factor analysis? (c) What is the internal consistency of the BFNE total and subscale scores? (d) Do patients with social anxiety disorder obtain more extreme BFNE total and subscale scores than do nonanxious control participants? (e) Do the BFNE total score and

\footnotetext{
${ }^{1}$ Findings regarding the psychometric properties of a variation of the BFNE Scale in patients with social anxiety disorder (Collins, Westra, Dozois \& Stewart, 2005) became available after the current study was conducted. On the basis of the findings of Rodebaugh et al. (2004), these authors modified the scale so that all 12 items were straightforwardly worded. Collins et al. (2005) reported that the modified version of the scale has good psychometric properties among patients with social anxiety disorder and that socially anxious patients achieve higher scores than do patients with panic disorder. Because of the modifications made to several items, the findings of Collins et al. are not directly comparable to those reported herein nor directly applicable to other research that has used the BFNE in the form provided by Leary (1983a). However, further research on this version of the BFNE appears to be warranted.
} 
subscale scores correlate with self-report and clinicianadministered measures of social anxiety? (f) Do the BFNE total score and subscale scores correlate more strongly with measures of social anxiety than with measures of depression, anxiety sensitivity, or worry? (g) Are the BFNE total score and subscale scores sensitive to treatment effects, and how does their sensitivity compare with that previously demonstrated by the original FNE?

\section{Method}

\section{Participants}

The majority of participants in the present study were patients who were evaluated at the Adult Anxiety Clinic of Temple University (AACT; $n=$ 90); the Center for Stress and Anxiety Disorders of the University at Albany, State University of New York (CSAD; $n=22)$; or the Anxiety Disorders Clinic of the New York State Psychiatric Institute (NYSPI; $n=$ 53); and who received a principal diagnosis of social anxiety disorder based on the criteria specified in the Diagnostic and Statistical Manual of Mental Disorders (4th ed. [DSM-IV]; American Psychiatric Association, 1994) (see the Semistructured Diagnostic Interviews section below for a detailed description of the interview process). Exclusion criteria and assessment procedures were similar across sites, with some variation due to established differences that preceded this study (see below). The majority of patients $(66.1 \%)$ were diagnosed with generalized social anxiety disorder. Patients with principal diagnoses other than social anxiety disorder or with comorbid diagnoses of schizophrenia, clinically significant depression that was associated with prominent risk of self-harm, substance abuse, or dependence in the past 6 months, or an organic mental disorder were excluded from the study. Patients with a current diagnosis of major depressive disorder were excluded at NYSPI but not at the other sites.

Data were also provided by nonanxious individuals $(n=32)$ who were recruited from the greater Philadelphia community and compensated for their participation. Nonanxious control participants were selected so as to reflect the composition of the AACT patient sample on age, race, and sex. Nonanxious participants also participated in a semistructured diagnostic interview but did not meet criteria for any current Axis I diagnosis other than, in one case, specific phobia. Although nonanxious participants completed a number of self-report measures, only their BFNE scores were used in the present study.

Patient samples from the three clinics were equivalent in terms of sex ratio, $\chi^{2}(2, N=164)=1.92, p=.38$; marital status, $\chi^{2}(2, N=163)=$ $1.04, p=.60$; education level, $\chi^{2}(1, N=163)=1.13, p=.57$; religion, $\chi^{2}(1, N=112)=2.12, p=.15 ;$ age, $F(2,160)=0.43, p=.65 ;$ and income, $F(1,54)=0.13, p=.73$. However, the three samples differed in race, $\chi^{2}(2, N=163)=22.06, p=.001$. The AACT sample differed from the CSAD sample, $\chi^{2}(1, N=111)=5.83, p=.02$, and both of these samples differed from the NYSPI sample, $\chi^{2}(1, N=141)=11.12, p=$ .001 , and $\chi^{2}(1, N=74)=17.94, p=.001$, respectively. Specifically, Caucasians were relatively overrepresented in the AACT sample and relatively underrepresented in the NYSPI sample. However, race was not associated with pretreatment BFNE scores across the clinical samples, $F(2$, $162)=0.47, p=.63$. Because the three clinical samples differed on only one variable and that variable was not related to BFNE scores, they were pooled into a larger group for the primary analyses. This larger clinical sample did not differ from the nonanxious control participants on sex ratio, $\chi^{2}(1, N=196)=0.06, p=.81$; marital status, $\chi^{2}(1, N=195)=1.09, p=$ .30 ; race, $\chi^{2}(1, N=195)=0.08, p=.78$; education level, $\chi^{2}(1, N=$ $195)=0.60, p=.44$; religion, $\chi^{2}(1, N=144)=0.79, p=.37$; age, $F(1$, $193)=0.13, p=.72$; or income, $F(1,86)=0.03, p=.86$. See Table 1 for a comparison of social anxiety disorder patients and nonanxious control participants on demographic characteristics.

\section{Measures}

All participants completed the BFNE. In addition, patients completed a series of measures that allowed for the assessment of convergent and discriminant validity of the BFNE.

\section{Semistructured Diagnostic Interviews}

The Anxiety Disorders Interview Schedule for DSM-IV—Lifetime Version (ADIS-IV-L; DiNardo, Brown, \& Barlow, 1994) provides probes and questions that assist in assigning $D S M-I V$ diagnoses for a subset of

Table 1

Comparison of Patients and Nonanxious Controls on Demographic Variables

\begin{tabular}{|c|c|c|c|c|c|c|c|c|}
\hline \multirow[b]{2}{*}{ Variable } & \multicolumn{4}{|c|}{ Patients $(n=165)$} & \multicolumn{4}{|c|}{ Controls $(n=32)$} \\
\hline & $n$ & $\%$ & $M$ & $S D$ & $n$ & $\%$ & $M$ & $S D$ \\
\hline \multicolumn{9}{|l|}{ Sex } \\
\hline Female & 68 & 41.2 & & & 14 & 43.8 & & \\
\hline Male & 97 & 58.8 & & & 18 & 56.3 & & \\
\hline \multicolumn{9}{|l|}{ Marital status } \\
\hline Single (never married) & 119 & 72.0 & & & 30 & 93.8 & & \\
\hline Married/once married & 46 & 28.0 & & & 2 & 6.2 & & \\
\hline \multicolumn{9}{|l|}{ Race } \\
\hline Caucasian & 106 & 64.2 & & & 20 & 62.5 & & \\
\hline Non-Caucasian & 59 & 35.8 & & & 12 & 37.5 & & \\
\hline \multicolumn{9}{|l|}{ Education } \\
\hline No college & 32 & 19.6 & & & 6 & 18.7 & & \\
\hline At least some college & 131 & 80.4 & & & 26 & 81.3 & & \\
\hline \multicolumn{9}{|l|}{ Religion } \\
\hline Catholic & 41 & 36.6 & & & 9 & 28.12 & & \\
\hline Non-Catholic & 71 & 63.4 & & & 23 & 71.88 & & \\
\hline Age (years) & & & 32.39 & 10.55 & & & 33.12 & 10.86 \\
\hline Income (thousands) & & & 27.15 & 30.89 & & & 25.98 & 25.82 \\
\hline
\end{tabular}

Note. Religion and income were assessed only for patients from the Adult Anxiety Clinic of Temple University and the Center for Stress and Anxiety Disorders of the University at Albany, State University of New York. 
psychiatric disorders, which include anxiety disorders, depressive disorders, and substance use disorders. The ADIS-IV-L was administered to all patients at the AACT and the CSAD and to all nonanxious control participants. Training criteria outlined by T. A. Brown, DiNardo, Lehman, and Campbell (2001) were satisfied by all AACT and CSAD interviewers in the present study. These criteria entailed studying the users' guide and observing at least three live interviews conducted by a senior interviewer. During live interviews, trainees made ratings and assigned diagnoses, which were later discussed and compared with those of the senior interviewer. Subsequently, trainees conducted at least three interviews in the presence of a senior interviewer. Trainees conducted these interviews while the senior clinician observed and interjected when warranted. Trainees and senior interviewers derived independent diagnoses. Prior to acting as independent interviewers, trainees had to match with a senior interviewer on three consecutive interviews on the principal diagnosis and on the presence of all additional current diagnoses. T. A. Brown et al. (2001) reported a kappa of .77 for a principal diagnosis of social anxiety disorder in a sample of 362 anxiety disorder patients who received two independent ADIS-IV-L interviews. Furthermore, all 112 patients from the current study who completed the ADIS-IV-L were also interviewed by a second assessor who administered the social anxiety disorder module of the ADIS-IV-L, and $100 \%$ agreement with the original principal diagnosis of social anxiety disorder was obtained $(\kappa=1.0)$.

The Structured Clinical Interview for $D S M-I V$ Axis I disorders-Patient Edition (SCID-I/P; First, Spitzer, Gibbon, \& Williams, 1996) is a widely used instrument that also provides $D S M-I V$ diagnoses and was administered at NYSPI. Training in the administration of the SCID-I/P was conducted in a manner similar to that of the ADIS-IV-L. Ventura, Liberman, Green, Shaner, and Mintz (1998) reported good agreement on symptoms $(\kappa=.76)$ and very good diagnostic accuracy $(83 \%)$ over a period of 5 years in a general clinical sample, when compared with trained diagnostic interviewers who viewed the interviews live or via videotape. However, interrater agreement of SCID-I/P diagnoses was not assessed in this study.

\section{Measures for the Assessment of Social Anxiety (Convergent Validity)}

Because fear of negative evaluation is the core feature of social anxiety disorder, convergent validity would be demonstrated if the BFNE were to be significantly correlated with the following measures of social anxiety and avoidance:

Social Phobia Scale (SPS; Mattick \& Clarke, 1998) and Social Interaction Anxiety Scale (SIAS; Mattick \& Clarke, 1998). The SPS is a measure of anxiety associated with being observed by others, and the SIAS is a measure of anxiety in dyads and groups. Both scales consist of 20 items, ${ }^{2}$ which are scored on a 5-point Likert-type rating scale ranging from 0 (not at all characteristic or true of me) to 4 (extremely characteristic or true of me). The following are examples of SPS items: "I feel selfconscious if I have to enter a room where others are already seated" and "I can get tense when I speak in front of other people." The following are sample SIAS items: "I find it easy to make friends of my own age" and "When mixing in a group, I find myself worrying I will be ignored." The SPS and the SIAS have demonstrated strong internal consistency in both clinical and undergraduate samples (all $\alpha \mathrm{s}>.86$ [SPS] and .84 [SIAS]; Heimberg, Mueller, Holt, Hope, \& Liebowitz, 1992; Mattick \& Clarke, 1998), and strong test-retest correlations have been reported in a sample of patients with social anxiety disorder at 4 and 12 weeks (all $r \mathrm{~s}>.91$, all $p s<.001 ;$ Mattick \& Clarke, 1998). Scores on the SPS and SIAS adequately discriminate individuals with social anxiety disorder from individuals with other anxiety disorders and from control participants. Furthermore, number of feared social interaction situations is more highly correlated with scores on the SIAS than with scores on the SPS, whereas number of reported feared performance situations is more highly correlated with scores on the SPS (E. J. Brown et al., 1997).

Fear Questionnaire-Social Phobia subscale (FQ-S; Marks \& Matthews, 1979). The FQ-S is a self-report questionnaire designed to assess avoidance of feared social situations and comprises five items scored on a 9-point Likert-type rating scale, ranging from 0 (would not avoid it) to 8 (always avoid it). Example items include "being watched or stared at" and "being criticized." The FQ-S demonstrates acceptable internal consistency (all $\alpha \mathrm{s}>$.60) in both clinical and undergraduate samples (Cox, Swinson, \& Shaw, 1991; Marks \& Matthews, 1979; Stanley, Beck, \& Zebb, 1996) and good 1-week test-retest reliability $(r=.81)$ in an undergraduate sample (Stanley et al., 1996). Furthermore, patients with social anxiety disorder score higher on the FQ-S than do those with panic disorder and agoraphobia (Cox et al., 1991).

Liebowitz Social Anxiety Scale (LSAS; Liebowitz, 1987). The 24-item LSAS is a clinician-administered measure that assesses fear and avoidance in social interaction (11 items) and performance (13 items) situations. Items are rated on separate 4-point Likert-type scales of fear and avoidance, which range from 0 (none and never, respectively) to 3 (severe and usually, respectively). The LSAS yields a total score, as well as separate scores for fear and avoidance of social interaction and situations. Because no hypotheses of the present study related to the distinction between fear and avoidance, only the total score was used. Example items include "talking to people in authority" and "entering a room when others are already seated." The LSAS demonstrates good internal consistency ( $\alpha=$ .96; Heimberg et al., 1999) and correlates significantly with scores on other measures of social anxiety (Heimberg et al., 1992).

\section{Measures for the Assessment of Other Constructs (Discriminant Validity)}

Participant responses to the following measures were available in our archive and were used to examine the discriminant validity of the BFNE. Discriminant validity would be demonstrated if lower correlations were exhibited between the BFNE and these measures:

Anxiety Sensitivity Index (ASI; Reiss, Peterson, Gursky, \& McNally, 1986). The ASI is a measure of fear of anxiety- and panic-related bodily sensations and the extent to which they are regarded as having catastrophic consequences. This measure comprises 16 items scored on a 5-point Likert-type rating scale, ranging from 0 (agree very little) to 4 (agree very much). Examples of ASI items include the following: "It scares me when my heart beats rapidly" and "Unusual body sensations scare me." The ASI demonstrates good internal consistency across both clinical and undergraduate control samples (all $\alpha \mathrm{s}>$.81) (Peterson \& Plehn, 1999) and good test-retest reliability with undergraduate samples for periods of 2 weeks $(r=.75)$ and as long as 3 years $(r=.71)$. Furthermore, panic symptoms are more accurately predicted by ASI scores than by trait anxiety (Peterson \& Plehn, 1999). A three-factor structure, which includes a social concerns factor, has been reported (Rodriguez, Bruce, Pagano, Spencer, \& Keller, 2004; Zinbarg, Barlow, \& Brown, 1997). However, because this structure has not been consistent across samples (Cox, Enns, Walker, Kjernisted, \& Pidlebury, 2001; Cox, Parker, \& Swinson, 1996) and the ASI was included in the present study as a measure of the discriminant validity of the BFNE, only the relationships between the ASI total score and the BFNE scores were examined.

Penn State Worry Questionnaire (PSWQ; Meyer, Miller, Metzger, \& Borkovec, 1990). The PSWQ is a self-report assessment of the frequency, excessiveness, and uncontrollability of worry. It consists of 16 items scored

${ }^{2}$ Two versions of the SIAS are currently available, one with 19 items, the other with 20 items. Responses to the 20 -item version, which has been much more commonly used in published research, were used in the present study. 
on a 5-point Likert-type scale ranging from 1 (not at all typical) to 5 (very typical). Examples of PSWQ items include the following: "Many situations make me worry" and "I am always worrying about something." The PSWQ demonstrates good internal consistency in both clinical and undergraduate samples (all $\alpha \mathrm{s}>.86$ ) and excellent 1-month test-retest reliability in undergraduate samples $(r=.93)$ (T. A. Brown, Antony, \& Barlow, 1992; Meyer et al., 1990). Furthermore, patients with generalized anxiety disorder (GAD) obtain higher PSWQ scores than do normal control participants and patients with other anxiety disorders, including social anxiety disorder (T. A. Brown et al., 1992).

BDI (Beck, Rush, Shaw, \& Emery, 1979). The BDI is a self-report scale that yields a single total score of depression. It comprises 21 items scored on a 4-point scale. Examples of the areas addressed by the items of the BDI include "loss of pleasure," with responses ranging from 0 (I get as much pleasure as I ever did from the things I enjoy) to 3 (I cannot get any pleasure from the things I used to enjoy), and "suicidal thoughts or wishes," with responses ranging from 0 (I do not have any thoughts of killing myself) to 3 (I would kill myself if I had the chance). A meta-analysis of responses to the BDI suggests that it exhibits good internal consistency in both clinical $(\alpha=.86)$ and nonclinical $(\alpha=.81)$ samples, and that it demonstrates acceptable test-retest reliability (all $r \mathrm{~s}>.62$, ranging from hours to months) in undergraduate, adult, and clinical samples (Beck, Steer, \& Garbin, 1988). Furthermore, BDI responses adequately discriminate depressed patients from control participants and from individuals with anxiety disorders (Beck et al., 1988). In addition, Coles, Gibb, and Heimberg (2001) demonstrated that the BDI correlates significantly more strongly with other measures of depression than with measures of either social anxiety or anxiety sensitivity among patients with social anxiety disorder.

\section{Procedure}

Patients completed the ADIS-IV-L or the SCID-I/P, the LSAS, and the battery of self-report questionnaires before the initiation of treatment. Treatment consisted primarily of cognitive-behavioral therapy (CBT) in either group $(n=72)$ or individual $(n=26)$ format. Other patients received either the monoamine oxidase inhibitor phenelzine $(n=30)$, phenelzine combined with CBGT $(n=19)$, or pill placebo $(n=18)$ as part of a controlled trial (Heimberg, 2002). Nonanxious control participants completed the questionnaires after the ADIS-IV-L interview and were compensated for their time. The ASI was not administered to patients receiving individual CBT.

Only participants who responded to all BFNE items at pretreatment were included in the present study. Furthermore, only participants who received and completed CBGT were included in treatment sensitivity analyses. This a priori decision was made because we were most specifically interested in comparing the treatment sensitivity of the BFNE with that of the FNE (as represented in the published literature). However, no study to date has examined the sensitivity of the FNE to individual CBT, and we could find only a single study that examined the effect of pharmacotherapy on FNE scores (Davidson et al., 1993). Furthermore, the subsamples of patients in the present study who received individual CBT $(n=26)$ or phenelzine $(n=30)$ were substantially smaller than the subsample that received CGBT $(n=72)$ and would provide less stable estimates of effect size.

CBGT was administered to groups of 5 or 6 patients by two therapists, over the course of 12 2.5-hr weekly sessions. Patients were presented with a cognitive-behavioral model of social anxiety disorder and trained in cognitive restructuring skills (identifying maladaptive automatic thoughts, disputing logical errors in these automatic thoughts, and developing rational responses). Furthermore, patients completed exposures to simulated feared situations during sessions and used cognitive restructuring skills before, during, and after homework assignments for in vivo exposure to feared situations. For a more detailed description of CBGT for social anxiety disorder, see Heimberg and Becker (2002) and Turk, Heimberg, and Hope (2001).

\section{Analysis Strategy}

In the context of the research questions outlined earlier, (a) confirmatory factor analysis was first performed, allowing an examination of the factor structure of the BFNE in patients with social anxiety disorder. On the basis of the factor analysis, (b) score distributions and (c) internal consistency of the BFNE total and subscale scores were examined. (d) An analysis of variance (ANOVA) was performed to determine whether patients with social anxiety disorder obtained more extreme BFNE total and subscale scores than did nonanxious control participants. The relative strength of the correlations exhibited between the BFNE total and subscale scores and the (e) social anxiety and (f) depression, anxiety sensitivity, and worry measures was examined to assess convergent and discriminant validity of the BFNE scores, respectively. Last, (g) effect sizes were calculated to examine the sensitivity of the BFNE total and subscale scores to treatment effects.

\section{Results}

Means and standard deviations for patients' and controls' responses to the questionnaire measures and the LSAS are displayed in Table 2.

\section{Confirmatory Factor Analysis}

Only the patient sample was included in the confirmatory factor analysis $(n=165)$. According to Bentler and Chou (1987), a ratio of 10 participants to every free parameter in a model is adequate to obtain a trustworthy solution in confirmatory factor analysis. A maximum of 16 parameters was established in the models tested, suggesting that our sample size was sufficient. On the basis of the findings of Rodebaugh et al. (2004), a confirmatory structural model with the 12 BFNE items serving as indicators of two correlated latent factors was tested and compared with a singlefactor model (by using the structural equation modeling software program AMOS 5; Arbuckle, 2002). The first factor of the experimental model consisted of the 8 straightforwardly worded BFNE items; the second factor consisted of the 4 reverse-scored items.

Table 2

Means (and Standard Deviations) on Study Measures for Social Anxiety Disorder Patients Before Treatment and for Nonanxious Controls

\begin{tabular}{|c|c|c|c|c|}
\hline \multirow[b]{2}{*}{ Measure } & \multicolumn{2}{|c|}{$\begin{array}{c}\text { Patients } \\
\text { (pretreatment) }\end{array}$} & \multicolumn{2}{|c|}{$\begin{array}{c}\text { Nonanxious } \\
\text { controls }\end{array}$} \\
\hline & $M$ & $S D$ & $M$ & $S D$ \\
\hline \multicolumn{5}{|l|}{ Brief Fear of Negative } \\
\hline Evaluation Scale & 46.91 & 9.27 & 26.81 & 4.78 \\
\hline Liebowitz Social Anxiety Scale & 74.39 & 22.75 & 14.32 & 9.66 \\
\hline Social Interaction Anxiety Scale & 49.21 & 15.45 & 13.69 & 11.14 \\
\hline Social Phobia Scale & 33.88 & 15.64 & 5.97 & 7.39 \\
\hline \multicolumn{5}{|l|}{ Fear Questionnaire-Social } \\
\hline Phobia subscale ${ }^{a}$ & 22.36 & 7.87 & - & - \\
\hline Anxiety Sensitivity Index & 26.93 & 13.20 & 8.41 & 7.46 \\
\hline Beck Depression Inventory & 12.81 & 8.61 & 1.47 & 1.93 \\
\hline Penn State Worry Questionnaire & 60.13 & 13.54 & 31.00 & 9.37 \\
\hline
\end{tabular}

Note. Sample size for patients varies from 138 to 165 as a result of missing data.

${ }^{\text {a }}$ Dashes indicate that the statistic was not computed because this scale was not administered to the control group. 
The items that are reverse-scored in the standard scoring of the BFNE were not reverse-scored for the current factor analysis. However, standard scoring was used in all other analyses included in this report.

The two-factor model yielded a significant chi-square statistic, $\chi^{2}(53, N=165)=112.27, p<.001$, as did the single-factor model, $\chi^{2}(54, N=165)=256.15, p<.001$, indicating a lessthan-perfect fit for both models. However, the chi-square statistic is highly sensitive to sample size and may overstate the lack of fit of a structural model (Bollen, 1989). Hu and Bentler (1999) have suggested cutoffs for alternative fit indices for evaluating the results of confirmatory factor analyses. Specifically, a cutoff of .95 or above on either the Tucker-Lewis Index (TLI; Tucker \& Lewis, 1973) or the comparative fit index (CFI; Bentler, 1990) combined with either a root-mean-square error of approximation (RMSEA) "close to .06" (Hu \& Bentler, 1999, p. 1) or a standardized root-mean-square residual (SRMR) "close to .08" (Hu \& Bentler, 1999, p. 1) provides a good combination of fit indices to conclude that a structural model has adequate or better fit to the data. Accordingly, the two-factor solution demonstrated adequate fit $(\mathrm{CFI}=.95 ; \mathrm{TLI}=.94 ;$ RMSEA $=.08 ;$ SRMR $=.04)$ and provided a better fit, $\chi^{2}(1, N=165)=143.88, p<.001$, to the data than did the one-factor solution $(\mathrm{CFI}=.82$; TLI $=.78$; RMSEA $=.15$; SRMR $=.44$ ). The path diagram for the twofactor model is displayed in Figure 1. Loadings for this model, which ranged from 0.61 to 0.87 on Factor 1 and from 0.69 to 0.86 on Factor 2, are displayed in Table 3.

Both subscales derived from the BFNE factors were highly correlated with the BFNE total score $(r=.91$ for Subscale 1 and $r=-.72$ for Subscale 2). Often, when factor-based subscale scores are highly correlated with one another, a higher order factor may be present. In the present study, the eight-item subscale based on Factor 1 (henceforth referred to as the Straightforward BFNE subscale [BFNE-S]) and the four-item subscale based on Factor 2 (henceforth referred to as the Reverse-scored BFNE subscale [BFNE-R]) were only moderately correlated $(r=-.42)$, suggesting the absence of a higher order factor. Several additional analyses were performed to evaluate alternative models but did not improve on the fit of the correlated two-factor model presented here. $^{3}$

\section{Psychometric Properties of the BFNE Total and Subscale} Scores

\section{Distribution of BFNE Total and Subscale Scores}

BFNE total score. The distribution of pretreatment BFNE total scores $(M=46.91, S D=9.27)$ was unimodal symmetric for social anxiety disorder patients (skewness $=-0.62, S E=.19$; kurtosis $=-0.40, S E=.38$ ) but leptokurtic for nonanxious control participants (skewness $=-0.39, S E=.41$; kurtosis $=1.79, S E=$ .81). BFNE total scores upon completion of CBGT $(M=37.15$, $S D=9.18$ ) maintained a unimodal symmetric distribution (skewness $=-0.03, S E=.33$; kurtosis $=-0.72, S E=.64$ ).

$B F N E-S$. The distribution of pretreatment BFNE-S scores $(M=30.60, S D=6.94)$ was unimodal symmetric for social anxiety disorder patients (skewness $=-0.78, S E=.19$; kurtosis $=0.19, S E=.38$ ) but positively skewed and leptokurtic for nonanxious control participants (skewness $=1.16, S E=.41$; kurtosis $=1.19, S E=.81)$. BFNE-S scores maintained a unimodal symmetric distribution (skewness $=-0.18, S E=.33$; kurtosis $=$ $-0.67, S E=.64)$ after CBGT $(M=21.89, S D=7.10)$.

$B F N E-R$. The distribution of pretreatment BFNE-R scores $(M=16.31, S D=4.10)$ was negatively skewed and leptokurtic for social anxiety disorder patients (skewness $=-1.25, S E=.19$; kurtosis $=1.04, S E=.38$ ) and slightly leptokurtic for nonanxious control participants (skewness $=-0.61, S E=.41$; kurtosis $=$ $0.82, S E=.81)$. The distribution of BFNE-R scores was, however, unimodal symmetric (skewness $=-0.23, S E=.33$; kurtosis $=$ $-0.76, S E=.64)$ following CBGT $(M=15.26, S D=3.43)$.

\section{Internal Consistency}

BFNE total score. Patient responses to the BFNE exhibited excellent internal consistency $(\alpha=.89)$. The internal consistency of the responses of nonanxious control participants was marginally adequate $(\alpha=.67)$, but internal consistency was good for the overall sample $(\alpha=.81)$.

$B F N E-S$. The BFNE-S demonstrated excellent internal consistency in the patient and control samples, as well as in the overall sample $(\alpha=.92, .90$, and .96 , respectively).

$B F N E-R$. The BFNE-R demonstrated strong internal consistency in the clinical sample $(\alpha=.85)$ and in the overall sample ( $\alpha=.84$ ) but marginally adequate internal consistency in the control sample ( $\alpha=.67)$. These figures are somewhat lower than demonstrated for the BFNE-S. However, Cronbach's alpha is sensitive to sample size, and a shorter scale may exhibit lower internal consistency than longer scales, regardless of the shorter scale's validity. Thus, it remains important to examine the other psychometric properties of the BFNE-R.

\section{Group Differences on BFNE Total and Subscale Scores}

BFNE total score. A one-way ANOVA revealed that social anxiety disorder patients scored significantly higher $(M=46.91$, $S D=9.27)$ on the BFNE than did nonanxious control participants $(M=26.81, S D=4.78), F(1,195)=142.71, p<.001$.

$B F N E-S$. A one-way ANOVA revealed that social anxiety disorder patients also scored significantly higher $(M=30.60$,

\footnotetext{
${ }^{3}$ Two additional analyses were conducted to examine alternative factor solutions. First, we fit an additional model in which the correlation between BFNE-S and BFNE-R was constrained to zero. The orthogonal (uncorrelated) model provided an inadequate fit to the data, $\chi^{2}(54, N=165)=$ $137.73, p<.001(\mathrm{CFI}=.93 ; \mathrm{TLI}=.91 ; \mathrm{RMSEA}=.10 ; \mathrm{SRMR}=.17)$, that was significantly poorer than the oblique (correlated) two-factor model proposed by Rodebaugh et al. (2004) and replicated in the current study, $\chi^{2}(1, N=165)=25.46, p<.001$. The results of this analysis were inconclusive, however, regarding the presence of a higher order factor Second, a model was tested that specified all 12 BFNE items loading onto one factor and, in addition, that specified method effects arising from the 4 reverse-scored items of the BFNE loading onto a second method-effects factor (for a detailed example of this type of analysis, see Hazlett-Stevens, Ullman, \& Craske, 2004). Results suggest that the method-effects model fits the data roughly as well as the two-factor model proposed by Rodebaugh et al. (2004) and replicated here; fit indices for the methods-effects model, $\chi^{2}(50, N=165)=110.59, p<.001(\mathrm{CFI}=.95 ; \mathrm{TLI}=.93$ RMSEA $=.09$; SRMR $=.04)$. However, given the poor psychometric showing of BFNE-R in other analyses, the two-factor model of Rodebaugh et al. (2004) is to be preferred.
} 


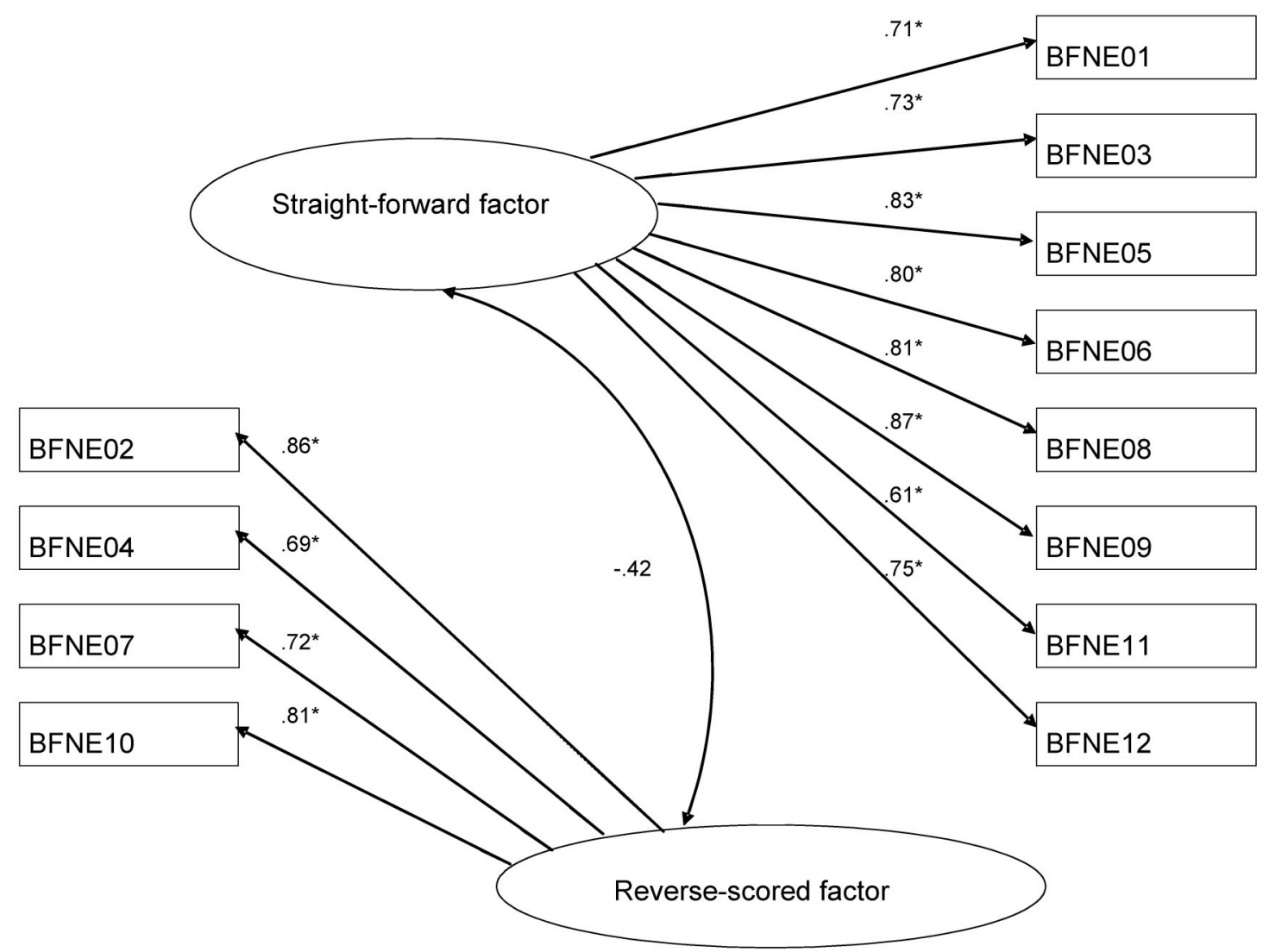

Figure 1. Completely standardized solution of hypothesized 2-factor model. BFNE $=$ Brief Fear of Negative Evaluation Scale. $* p<.05$.

$S D=6.94)$ on the BFNE-S than did nonanxious control participants $(M=12.50, S D=4.52), F(1,195)=200.69, p<.001$. It is noteworthy that the difference in average BFNE total scores between social anxiety disorder patients and the nonanxious control participants (20.10) is only slightly larger than the respective difference in BFNE-S scores (18.10); approximately $90 \%$ of the difference in BFNE total scores is attributable to the BFNE-S.

$B F N E-R$. A one-way ANOVA revealed that social anxiety disorder patients scored significantly higher $(M=16.31, S D=$ 4.10) on BFNE-R than did nonanxious control participants $(M=$ $14.31, S D=3.00), F(1,195)=6.85, p=.01$.

\section{Convergent Validity}

BFNE total score. A Bonferroni correction $(.05 / 4=.0125)$ was applied, which controlled for the number of comparisons between each of the BFNE scores and the four social anxiety measures. Upon applying the Bonferroni correction, we found that the BFNE total was significantly correlated with all measures of social anxiety (see Table 4).

$B F N E-S$. After Bonferroni correction, the BFNE-S was significantly correlated with all measures of social anxiety.

$B F N E-R$. After Bonferroni correction, the BFNE-R correlated modestly but significantly with the LSAS. It was significantly correlated with none of the other measures of social anxiety.
We also examined whether any of the three BFNE scores related more strongly to the various social anxiety measures. Significance tests were conducted by using a Fisher's $r$-to- $z$ transformation refined by Meng, Rosenthal, and Rubin (1992). A Bonferroni correction $(.05 / 4=.0125)$ was applied. The BFNE total score and BFNE-S related equivalently to three of the social anxiety measures; however, BFNE-S was more highly correlated than the BFNE total score to the SIAS $(p<.001)$. Furthermore, both the BFNE and the BFNE-S were more highly correlated than the BFNE-R to all social anxiety measures (all $p$ s $<.001)$.

\section{Discriminant Validity}

BFNE total score. A Bonferroni correction $(.05 / 3=.0167)$ was applied. The BFNE total was significantly correlated with all three discriminant measures (see Table 5). Notably, the magnitude of the relationship reported by Saluck et al. (2000) between the BFNE and the BDI $\left(r=.61 ; r^{2}=.372\right)$ in a small sample of social anxiety disorder patients was almost four times that obtained in the present study $\left(r=.31 ; r^{2}=.096\right)$.

Significance tests were conducted to determine whether BFNE scores correlated more strongly with measures of social anxiety than with measures of anxiety sensitivity, depression, or worry. These tests were conducted according to Meng et al. (1992). A Bonferroni correction $(.05 / 12=.004)$ was applied, 
Table 3

Factor Analysis of the Brief Fear of Negative Evaluation Scale

\begin{tabular}{llll}
\hline \multicolumn{1}{c}{ Item } & $f^{*}$ & $f$ & $S E$ \\
\hline \multicolumn{1}{c}{ Straightforwardly worded factor } & & & \\
& & & \\
& .87 & 1.24 & .12 \\
& .83 & 1.31 & .13 \\
9. I am usually worried about what kind of impression I make. & .81 & 1.28 & .13 \\
5. I am afraid that others will not approve of me. & .80 & 1.22 & .12 \\
8. When I am talking with someone, I worry about what they may be thinking about me. & .75 & 1.11 & .12 \\
6. I am afraid that people will find fault with me. & .73 & 1.00 & .11 \\
12. I often worry that I will say or do the wrong things. & & & \\
3. I am frequently afraid of other people noticing my shortcomings. & .71 & 1.00 & - \\
1. I worry about what people will think of me even when I know it doesn't make any & .61 & 0.98 & .13 \\
difference. & & &
\end{tabular}

Reverse-worded factor

2. I am unconcerned even if I know people are forming an unfavorable impression of me. $\quad .86 \quad 1.00 \quad-$

10. If I know someone is judging me, it has little effect on me. $\quad \begin{array}{llll}.03 & 1.03 & .09\end{array}$

7. Other people's opinions of me do not bother me. $\quad \begin{array}{llll}72 & 0.82 & .08\end{array}$

$\begin{array}{llll}\text { 4. I rarely worry about what kind of impression I am making on someone. } & .72 & 0.89 & .10\end{array}$

Note. Items are ordered by magnitude of $f^{*}$ per factor. Dashes indicate that the statistic was not computed because the item was used to scale the factor. $f^{*}=$ standardized factor loading; $f=$ unstandardized factor loading. The items are from "A Brief Version of the Fear of Negative Evaluation Scale," by M. R. Leary, Personality and Social Psychology Bulletin, 9, p. 373, copyright 1983. Reprinted by permission of Sage Publications.

which controlled for the number of comparisons of each of the four social anxiety measures to each of the three discriminant validity measures $(4 \times 3=12)$. The BFNE total score was more strongly related to the LSAS than to the ASI or the BDI (all $p s<.001)$. Furthermore, the relationship between the BFNE and the LSAS remained robust upon controlling for anxiety sensitivity and depressive symptoms (both $p$ s $<.001$ ). In contrast, partial correlations of the BFNE with the ASI and BDI, controlling for social anxiety (LSAS scores), were no longer significant (all $p \mathrm{~s}>.08$ ). All other comparisons of the relative strength of correlations of the social anxiety measures, $\mathrm{ASI}$, and BDI to the BFNE total score were nonsignificant.

Table 4

Zero-Order Correlations of the Brief Fear of Negative Evaluation Scale and Its Factors With Social Anxiety Measures Before Treatment Among Patients With Social Anxiety Disorder

\begin{tabular}{lccc}
\hline \multicolumn{1}{c}{ Measure } & $\begin{array}{c}\text { BFNE } \\
\text { total score }\end{array}$ & BFNE-S & BFNE-R \\
\hline Liebowitz Social Anxiety Scale & $.56^{\mathrm{a}}$ & $.59^{\mathrm{a}}$ & $.26^{\mathrm{a}}$ \\
Social Interaction Anxiety Scale & $.38^{\mathrm{a}}$ & $.46^{\mathrm{a}}$ & .07 \\
$\begin{array}{l}\text { Social Phobia Scale } \\
\text { Fear Questionnaire-Social Phobia } \\
\quad \text { subscale }\end{array}$ & $.35^{\mathrm{a}}$ & $.40^{\mathrm{a}}$ & .11 \\
\hline
\end{tabular}

Note. Ns vary from 148 to 165 as a result of missing data. Bonferroni correction was calculated on the basis of the number of comparisons per column. BFNE $=$ Brief Fear of Negative Evaluation Scale; BFNE-S = BFNE subscale based on straightforwardly worded items; BFNE-R = BFNE subscale based on reverse-scored items.

${ }^{\mathrm{a}} p=(.05 / 4)=.0125$.
An unexpected finding was the strength of the correlation between the BFNE and the PSWQ. Indeed, the PSWQ correlated as strongly with the BFNE as did the LSAS, the SIAS, and the FQ-S, and more strongly with the BFNE than did the SPS $(p<.001)$. Furthermore, the relationship between the BFNE and the PSWQ remained robust (all $p$ s $<.001$ ) upon controlling separately for the presence of comorbid GAD, ${ }^{4}$ social anxiety (LSAS), anxiety sensitivity (ASI), and depression (BDI).

$B F N E-S$. Using the same approach described above, we found that BFNE-S was more strongly related to the LSAS than to the ASI or the BDI, and more strongly related to the SIAS than to the ASI (all $p \mathrm{~s}<.001$ ). Furthermore, the relationships between the BFNE-S and the LSAS and SIAS remained robust upon controlling for anxiety sensitivity and depressive symptoms (all $p \mathrm{~s}<.001$ ). In contrast, partial correlations of BFNE-S with the ASI and BDI controlling for social anxiety (LSAS scores) were no longer significant (all $p$ s $>.08$ ). All other comparisons of the relative strength of correlations of the social anxiety measures, ASI, and BDI to BFNE-S were nonsignificant.

BFNE-S was also strongly correlated with the PSWQ. Indeed, the PSWQ correlated as strongly with the BFNE-S as did all social anxiety measures. Furthermore, the relationship between the BFNE-S and the PSWQ remained robust (all $p$ s $<$

\footnotetext{
${ }^{4}$ Of the 112 participants treated at the AACT or the CSAD (approximately 19.6\%), 22 were assigned a comorbid diagnosis of GAD at intake. Comorbid diagnostic information was not available for patients treated at NYSPI.
} 
Table 5

Zero-Order Correlations of the Brief Fear of Negative

Evaluation Scale and Its Factors With Discriminant Measures Before Treatment Among Patients With Social Anxiety Disorder

\begin{tabular}{lccc}
\hline \multicolumn{1}{c}{ Measure } & $\begin{array}{c}\text { BFNE } \\
\text { total score }\end{array}$ & BFNE-S & BFNE-R \\
\hline Anxiety Sensitivity Index & $.27^{\mathrm{a}}$ & $.27^{\mathrm{a}}$ & .16 \\
Beck Depression Inventory & $.31^{\mathrm{a}}$ & $.32^{\mathrm{a}}$ & .15 \\
Penn State Worry Questionnaire & $.51^{\mathrm{a}}$ & $.51^{\mathrm{a}}$ & $.31^{\mathrm{a}}$ \\
\hline
\end{tabular}

Note. Ns vary from 138 to 160 as a result of missing data. Bonferroni correction was calculated on the basis of the number of comparisons per column. BFNE = Brief Fear of Negative Evaluation Scale; BFNE-S = BFNE subscale based on straightforwardly worded items; BFNE-R = BFNE subscale based on reverse-scored items.

${ }^{\mathrm{a}} p=(.05 / 3)=.0167$

.001) upon controlling separately for the presence of comorbid GAD, social anxiety (LSAS), anxiety sensitivity (ASI), and depression (BDI).

$B F N E-R$. Using the same approach described above, we found that the PSWQ related more strongly to the BFNE-R than did the SIAS $(p=.0035)$. All other comparisons of the relative strength of correlations between the BFNE-R and social anxiety and discriminant measures were nonsignificant. Recall that the BFNE-R correlated significantly with only the LSAS and the PSWQ.

The relationship between the BFNE-R and the PSWQ remained robust (all $p \mathrm{~s}<.003$ ) upon controlling separately for the presence of comorbid GAD, social anxiety (LSAS), anxiety sensitivity (ASI), and depression (BDI).

\section{Unique Predictive Ability of BFNE Subscales}

We computed separate hierarchical regression equations by using the BFNE-S and the BFNE-R as predictors and the LSAS as the criterion variable, in order to examine whether either of the BFNE subscales accounted for variance in social anxiety beyond that already accounted for by the other subscale score. In the first equation, we entered BFNE-S in the first step and BFNE-R in the second step. After the first step, BFNE-S was significantly and positively related to the LSAS $\left(R^{2}=.35\right), F(1,146)=78.43, p<.001$. BFNE-R did not significantly improve the prediction of LSAS scores $\left(\Delta R^{2}=.002\right)$, $\Delta F(1,145)=0.43, p=.52$. In the second equation, we entered BFNE-R in the first step and BFNE-S in the second step. After the first step, BFNE-R was significantly and positively related to the LSAS $\left(R^{2}=.07\right), F(1,146)=10.75, p<.001$. However, the BFNE-S significantly improved the prediction of LSAS scores, $\Delta R^{2}=.28, \Delta F(1,145)=63.21, p<.001$. Thus, only BFNE-S uniquely contributed to the prediction of social anxiety.

\section{Sensitivity to Treatment Effects}

Within-treatment effect sizes $(d)$ were calculated only for patients who completed CBGT $(n=54 ; 18$ of 72 patients dropped out), according to the following formula (Cohen, 1988):

$$
d=\left[\left(\mathrm{M}_{\text {Pretest }}-\mathrm{M}_{\text {Posttest }}\right) / \sqrt{\left(\mathrm{SD}_{\text {Pretest }}^{2}+\mathrm{SD}_{\text {Posttest }}^{2}\right) / 2}\right] .
$$

Confidence intervals (CI) for effect sizes were calculated according to the following formula (Cohn \& Becker, 2003):

$$
\mathrm{CI}=\left[d \pm 1.96 \sqrt{1 /\left(\left(\mathrm{SD}_{\text {Pretest }}^{2}+\mathrm{SD}_{\text {Posttest }}^{2}\right) / 2\right)}\right] .
$$

BFNE total scores. This analysis yielded a $d$ of $0.90(\mathrm{CI}=$ $0.68,1.11)$.

BFNE-S scores. The treatment effect size of BFNE-S was 1.08 $(\mathrm{CI}=0.80,1.36)$.

$B F N E-R$ scores. Cohen's $d$ was not calculated for BFNE-R because of its poor showing in the examination of its psychometric properties. Specifically, BFNE-R scores (a) were not normally distributed for social anxiety disorder patients before treatment, (b) did not relate significantly to the majority of the social anxiety measures, and (c) did not contribute unique variance to the prediction of social anxiety scores.

\section{Education and Response to the BFNE Total and Subscale Scores}

A parsimonious explanation of the finding that BFNE-S related more strongly to measures of social anxiety than did BFNE-R, originally put forth by Rodebaugh et al. (2004), is that the straightforwardly worded items perform better because they are less confusing to respondents. For example, socially anxious individuals are expected to respond to such BFNE-R descriptions as "Other people's opinions of me do not bother me" as being not at all characteristic of me, which constitutes a double negative. If this explanation is viable, then one might hypothesize that level of education would be more strongly related to BFNE-R scores than to BFNE-S scores. Individuals with some college education may have better reading skills and therefore be better able to interpret double negative constructions. In fact, BFNE-R scores were significantly lower in the subsample of patients who had no college education $(M=14.63, S D=4.23)$ than in the subsample of patients with at least some college education $(M=16.32, S D=$ $3.89), F(1,161)=7.06, p=.009 ; d=0.41$. In contrast, BFNE-S scores of patients who had no college education $(M=30.31, S D=$ 6.77) did not differ significantly from those who had at least some college education $(M=30.61, S D=7.02), F(1,161)=0.05, p=$ $.83 ; d=0.04$.

\section{Discussion}

The present study is the first to thoroughly examine the psychometric properties of the BFNE in a large sample of socially anxious patients. Several analyses were conducted that provide information about the structure, reliability, and validity of the BFNE total and subscale scores in this sample. First, confirmatory factor analyses supported the two-factor structure originally reported in undergraduate participants by Rodebaugh et al. (2004). Second, the distributions of BFNE and BFNE-S scores both before and after CBT were unimodally symmetric for social anxiety disorder patients. This was also the case for BFNE-R scores of social anxiety disorder patients after CBT, but these scores were negatively skewed and leptokurtic before treatment. The distribution of the nonanxious control responses was not unimodally symmetric for any of the BFNE scores. Third, patient responses to the BFNE total score, BFNE-S, and BFNE-R exhibited excellent internal consistency, although the internal consistency of the BFNE total score and BFNE-R was considerably lower in nonanxious control participants. All BFNE scores exhibited strong 
internal consistency in the combined sample. Fourth, social anxiety disorder patients obtained more extreme scores on the BFNE total score, BFNE-S, and BFNE-R than did nonanxious control participants. Fifth, the BFNE total score and BFNE-S correlated with all self-report and clinician-administered measures of social anxiety, whereas BFNE-R correlated with only one of the four measures of social anxiety. In addition, BFNE-S contributed unique variance to the prediction of social anxiety, but BFNE-R did not. Sixth, neither the BFNE total score nor BFNE-S correlated significantly with anxiety sensitivity or depressive symptoms when social anxiety was controlled. However, all BFNE indices correlated strongly with the measure of worry. Last, both the BFNE total score and BFNE-S appeared to be sensitive to the effects of CBGT for social anxiety disorder.

Confirmatory factor analysis supported Rodebaugh et al.'s (2004) two-factor solution; the four reverse-scored items formed a factor that separated from the eight straightforwardly worded items. Notably, a sizable body of literature demonstrates that when scales include a combination of straightforward and reverse-scored items, factor analyses frequently produce distinct factors based on this difference in item construction (e.g., Bagozzi, 1993; Bagozzi \& Heatherton, 1994; Baumgartner \& Steenkamp, 2001; T. A. Brown, 2003; Carmines \& Zeller, 1979; Marsh, 1992, 1996; Spector, Van Katwyk, Brannick, \& Chen, 1997). The extent to which this phenomenon has been studied raises the question of whether such factors should be considered meaningful or discarded as an expected artifact of method effects or response biases (T. A. Brown, 2003). However, the superiority of the present two-factor solution should be judged by the utility of subscales based on the straightforward and the reverse-scored factors, in addition to the specifics of results of the factor analyses. Several results favor the two-factor solution: The two BFNE subscale scores were only moderately correlated; BFNE-R scores were significantly lower among patients with no college education (i.e., they reported less fear of negative evaluation on these items) than among patients with at least some college education, whereas BFNE-S scores of these groups did not differ; and BFNE-S scores were more strongly correlated with measures of social anxiety than were BFNE-R scores. None of these outcomes would be expected if BFNE-S and BFNE-R were different expressions of the same underlying construct. Thus, it does not appear that the BFNE-R is a valid measure of fear of negative evaluation. Parenthetically, the present findings underscore the need for future research examining the psychometric implications of reverse-scored items in selfreport measures and the merits and drawbacks of their inclusion.

Overall, the BFNE total score and BFNE-S demonstrated comparable strong psychometric properties in patients with social anxiety disorder. However, the validity of BFNE-R may be seriously questioned, both by the education finding reported above and its failure to correlate with three of four measures of social anxiety. Analyses of distribution characteristics, internal consistency, group differences, and correlations with measures of social anxiety also suggest that the full BFNE provides little apparent psychometric advantage over BFNE-S; in fact, the results suggest a slight advantage for BFNE-S. Furthermore, it can reasonably be argued that because the full BFNE contains the demonstrably faulty BFNE-R items, BFNE-S is a more psychometrically valid scale and is therefore the better index for assessing fear of negative evaluation.
Both the full BFNE and BFNE-S appear to be sensitive to treatment-induced changes associated with CBGT. An issue raised earlier is whether the dimensional nature of the BFNE response options may provide heightened sensitivity to the effects of treatment, in comparison to the original true-false FNE scale. To address this issue, we obtained several estimates of the treatment sensitivity of the original FNE, by using Cohen's $d$, for comparison with those reported here for the BFNE and BFNE-S (the treatment sensitivity of the BFNE-R was not addressed in this comparison). First, Cox, Ross, Swinson, and Direnfeld (1998) reported on the effects of the same CBGT program used in the present study in a sample of 25 patients and achieved an effect size of 0.68 for the original FNE. Second, an effect size was calculated by using data collected from 93 participants who also received the same CBGT program, yielding an effect size of 0.45 (Heimberg, 2005). In these samples, the FNE demonstrated small to medium effects. In the present study, the effect sizes for CBGT for both the BFNE $(d=0.90)$ and BFNE-S $(d=1.08)$ were quite large and superior to those reported above for the FNE. In addition, ours is not the first examination of the treatment sensitivity of the BFNE in the literature. Taylor, Woody, McLean, and Koch (1997) reported an effect size $(d=0.79)$ smaller than those we obtained for the BFNE. However, their effect size was calculated after combining two treatment conditions, one of which included a theoretically inert control module, and the effect size for the active treatment condition alone was not reported.

Another important issue is the nature of the relationship between the BFNE and the PSWQ. Whereas the BFNE and BFNE-S appeared to be more strongly related to social anxiety than to anxiety sensitivity or depression, they were not more strongly related to social anxiety than to worry. Furthermore, this relationship was not attributable to the presence of comorbid GAD. Several explanations for the strength of this relationship exist. First, it has been previously demonstrated that PSWQ scores are elevated in patients with social anxiety disorder (although not to the extreme levels noted in patients with GAD; T. A. Brown et al., 1992), and this correlation may simply reflect elevated worry in socially anxious patients. Second, assessment devices do not necessarily measure the same construct in samples with different disorders. For example, it is possible that responses to the PSWQ among patients with social anxiety disorder pertain primarily to worry about social or performance demands or outcomes, whereas responses to the PSWQ among patients with GAD may tap into the more ruminative process of uncontrollable and excessive worry about a variety of life domains (e.g., finances, health, work, day-to-day matters). Many items of the PSWQ are ambiguous with respect to areas of worry and so could be construed as pertaining to social anxiety concerns. For example, the items "My worries overwhelm me" and "Once I start worrying, I cannot stop" (Meyer et al., 1990) could elicit responses from social anxiety patients that are grounded in the context of social situations. Although this possibility could not be addressed in the present study, it could be tested by examining whether PSWQ scores show different patterns of correlations in samples of patients with social anxiety disorder or GAD. For example, does the PSWQ correlate more highly with measures of social anxiety in patients with social anxiety disorder than in patients with GAD? Does it correlate more highly with measures of trait anxiety, tension, or stress in patients with GAD than it does among patients with social anxiety disorder? 
Several limitations to the present study exist. First, only patients with a principal diagnosis of social anxiety disorder and without comorbid diagnoses of schizophrenia, clinically significant depression that was associated with prominent risk of self-harm, substance abuse, or dependence in the past six months, or organic mental disorders were included in the present study. Also, the sample was a relatively well-functioning one. To illustrate, four fifths of the sample had at least some college education. Moreover, the present study did not examine the relationships between responses to the BFNE and the other measures in the control sample. Therefore, the utility of the BFNE with more severely impaired samples and with individuals without psychological disorders are topics for future research. Second, although the sensitivity of the BFNE to treatment effects was an important issue in the present article, only responses of patients treated with CBGT were included in the treatment sensitivity analysis. Future studies should examine the sensitivity of the BFNE to different treatments for social anxiety disorder, including drug treatments. Third, it is necessary to extend the validation of the BFNE through the inclusion of multimethod data sources. Although the BFNE relates as expected with self-report measures and at least one clinicianadministered measure of social anxiety, the comparison of BFNE responses with behavioral and physiological indicators is an essential next step in the examination of its construct validity.

In conclusion, the present findings suggest that the BFNE-S is a psychometrically sound index of fear of negative evaluation that possesses psychometric properties superior to those of BFNE-R. Furthermore, results favor use of the briefer BFNE-S over the full BFNE, if for no other reason than that the full BFNE contains the demonstrably faulty BFNE-R items. A scoring strategy that may be ideal for the BFNE, given the present findings, has been recommended by Marsh (1996), who suggested that a small number of reverse-scored items may be included in psychological measures to disrupt or evaluate potential response biases, but not used when scoring the measure. Overall, our findings indicate that the BFNE is a generally valid measure of fear of negative evaluation and therefore lend some credence to the validity of previous studies that have used the BFNE as a measure of fear of negative evaluation in similar samples (although the inclusion of reversescored items may have led to the underestimation of effect).

\section{References}

American Psychiatric Association. (1994). Diagnostic and statistical manual of mental disorders (4th ed.). Washington, DC: Author.

Arbuckle, J. L. (2002). AMOS for Windows. Analysis of moment structures (Version 5). Chicago: SmallWaters.

Asmundson, G. J. G., \& Stein, M. B. (1994). Selective processing of social threat in patients with generalized social phobia: Evaluation using a dot-probe paradigm. Journal of Anxiety Disorders, 8, 107-117.

Bagozzi, R. P. (1993). An examination of the psychometric properties of measures of negative affect in the PANAS-X scales. Journal of Personality and Social Psychology, 65, 836-851.

Bagozzi, R. P., \& Heatherton, T. F. (1994). A general approach to representing multifaceted personality constructs: Application to state selfesteem. Structural Equation Modeling, 1, 35-67.

Baumgartner, H., \& Steenkamp, J. B. E. M. (2001). Response styles in marketing research: A cross-national investigation. Journal of Marketing Research, 38, 143-156.

Beck, A. T., Rush, A. J., Shaw, B. F., \& Emery, G. (1979). Cognitive therapy of depression. New York: Guilford Press.
Beck, A. T., Steer, R. A., \& Garbin, M. G. (1988). Psychometric properties of the Beck Depression Inventory: Twenty-five years of evaluation. Clinical Psychology Review, 8, 77-100.

Bentler, P. M. (1990). Fit indexes, Lagrange multipliers, constraint changes and incomplete data in structural models. Multivariate Behavioral Research, 25, 163-172.

Bentler, P. M., \& Chou, C. (1987). Practical issues in structural modeling. Sociological Methods and Research, 16, 78-117.

Bollen, K. A. (1989). Structural equations with latent variables. New York: Wiley.

Brown, E. J., Turovsky, J., Heimberg, R. G., Juster, H. R., Brown, T. A., \& Barlow, D. H. (1997). Validation of the Social Interaction Anxiety Scale and the Social Phobia Scale across the anxiety disorders. Psychological Assessment, 9, 21-27.

Brown, T. A. (2003). Confirmatory factor analysis of the Penn State Worry Questionnaire: Multiple factors or method effects? Behaviour Research and Therapy, 41, 1411-1426.

Brown, T. A., Antony, M. M., \& Barlow, D. H. (1992). Psychometric properties of the Penn State Worry Questionnaire in a clinical anxiety disorders sample. Behaviour Research and Therapy, 30, 33-37.

Brown, T. A., DiNardo, P. A., Lehman, C. L., \& Campbell, L. A. (2001) Reliability of $D S M-I V$ anxiety and mood disorders: Implications for the classification of emotional disorders. Journal of Abnormal Psychology, 110, 49-58.

Carmines, E. G., \& Zeller, R. A. (1979). Reliability and validity assessment. Beverly Hills, CA: Sage.

Clark, D. M., \& Wells, A. (1995). A cognitive model of social phobia. In R. G. Heimberg, M. R. Liebowitz, D. A. Hope, \& F. R. Schneier (Eds.), Social phobia: Diagnosis, assessment, and treatment (pp. 69-93). New York: Guilford Press.

Cohen, J. (1988). Statistical power analysis for the behavioral sciences (2nd ed.). Hillsdale, NJ: Erlbaum.

Cohn, L. D., \& Becker, B. J. (2003). How meta-analysis increases statistical power. Psychological Methods, 8, 243-253.

Coles, M. E., Gibb, B. E., \& Heimberg, R. G. (2001). Psychometric evaluation of the Beck Depression Inventory in adults with social anxiety disorder. Depression and Anxiety, 14, 145-148.

Collins, K. A., Westra, H. A., Dozois, D. J. A., \& Stewart, S. (2005). The validity of the brief version of the Fear of Negative Evaluation Scale. Journal of Anxiety Disorders, 19, 345-359.

Cox, B. J., Enns, M. W., Walker, J. R., Kjernisted, K., \& Pidlebury, S. R. (2001). Psychological vulnerabilities in patients with major depression vs. panic disorder. Behaviour Research and Therapy, 39, 563-567.

Cox, B. J., Parker, J. A., \& Swinson, R. P. (1996). Anxiety sensitivity: Confirmatory evidence for a multidimensional construct. Behaviour Research and Therapy, 34, 591-598.

Cox, B. J., Ross, L., Swinson, R. P., \& Direnfeld, D. M. (1998). A comparison of social phobia outcome measures in cognitive-behavioral group therapy. Behavior Modification, 22, 285-297.

Cox, B. J., Swinson, R. P., \& Shaw, B. F. (1991). Value of the Fear Questionnaire in differentiating agoraphobia and social phobia. British Journal of Psychiatry, 159, 842-845.

Davidson, J. R. T., Potts, N., Richichi, E., Krishnan, R., Ford, S. M., Smith, R., \& Wilson, W. H. (1993). Treatment of social phobia with clonazepam and placebo. Journal of Clinical Psychopharmacology, 13, 423-428.

DiNardo, P. A., Brown, T. A., \& Barlow, D. H. (1994). Anxiety Disorders Interview Schedule for DSM-IV_Lifetime Version (ADIS-IV-L). San Antonio, TX: Psychological Corporation.

First, M. B., Spitzer, R. L., Gibbon, M., \& Williams, J. (1996). Structured Clinical Interview for DSM-IV Axis I disorders-Patient Edition (SCID$I / P$, Version 2.0). New York: New York State Psychiatric Institute.

Friend, R. M., \& Gilbert, J. (1973). Threat and fear of negative evaluation as determinants of locus of social comparison. Journal of Personality, $41,328-340$.

Hazlett-Stevens, H., Ullman, J. B., \& Craske, M. G. (2004). Factor structure of the Penn State Worry Questionnaire. Assessment, 11, 361-370. 
Heimberg, R. G. (1994). Cognitive assessment strategies and the measurement of outcome of treatment for social phobia. Behaviour Research and Therapy, 32, 269-280.

Heimberg, R. G. (2002, November). The understanding and treatment of social anxiety: What a long strange trip it's been (and will be). Presidential address presented at the annual meeting of the Association for Advancement of Behavior Therapy, Reno, NV.

Heimberg, R. G. (2005). [Sensitivity of the Fear of Negative Evaluation Scale to the effects of cognitive-behavioral group therapy for social anxiety disorder]. Unpublished raw data.

Heimberg, R. G., \& Becker, R. E. (2002). Cognitive-behavioral group therapy for social phobia: Basic mechanisms and clinical strategies. New York: Guilford Press.

Heimberg, R. G., Horner, K. J., Juster, H. R., Safren, S. A., Brown, E. J., Schneier, F. R., \& Liebowitz, M. R. (1999). Psychometric properties of the Liebowitz Social Anxiety Scale. Psychological Medicine, 29, 199-212.

Heimberg, R. G., Mueller, G. P., Holt, C. S., Hope, D. A., \& Liebowitz, M. R. (1992). Assessment of anxiety in social interaction and being observed by others: The Social Interaction Anxiety Scale and the Social Phobia Scale. Behavior Therapy, 23, 53-73.

Heinrichs, N., \& Hofmann, S. G. (2001). Information processing in social phobia: A critical review. Clinical Psychology Review, 21, 751-770.

Hope, D. A., Rapee, R. M., Heimberg, R. G., \& Dombeck, M. (1990). Representations of the self in social phobia: Vulnerability to social threat. Cognitive Therapy and Research, 14, 177-189.

Hu, L., \& Bentler, P. M. (1999). Cutoff criteria for fit indexes in covariance structure analysis: Conventional criteria versus new alternatives. Structural Equation Modeling, 6, 1-55.

Leary, M. R. (1983a). A brief version of the Fear of Negative Evaluation Scale. Personality and Social Psychology Bulletin, 9, 371-375.

Leary, M. R. (1983b). Understanding social anxiety: Social, personality, and clinical perspectives. Beverly Hills, CA: Sage.

Liebowitz, M. R. (1987). Social phobia. Modern Problems in Pharmacopsychiatry, 22, 141-173.

Lundh, L.-G., \& Sperling, M. (2002). Social anxiety and the post-event processing of socially distressing events. Cognitive Behaviour Therapy, 31, 129-134.

Mansell, W., \& Clark, D. M. (1999). How do I appear to others? Social anxiety and processing of the observable self. Behaviour Research and Therapy, 37, 419-434.

Marks, I. M., \& Matthews, A. M. (1979). Brief standard self-rating for phobic patients. Behaviour Research and Therapy, 17, 263-267.

Marsh, H. W. (1992). Self-Description Questionnaire III: Manual. Macarthur, New South Wales, Australia: Faculty of Education, University of Western Sydney.

Marsh, H. W. (1996). Positive and negative global self-esteem: A substantively meaningful distinction or artifactors? Journal of Personality and Social Psychology, 70, 810-819.

Mattick, R. P., \& Clarke, J. C. (1998). Development and validation of measures of social phobia scrutiny fear and social interaction anxiety. Behaviour Research and Therapy, 36, 455-470.

Meng, X. L., Rosenthal, R., \& Rubin, D. B. (1992). Comparing correlated correlation coefficients. Psychological Bulletin, 111, 172-175.

Meyer, T. J., Miller, M. L., Metzger, R. L., \& Borkovec, T. D. (1990). Development and validation of the Penn State Worry Questionnaire. Behaviour Research and Therapy, 28, 487-495.

Miller, R. S. (1995). On the nature of embarrassability: Shyness, social evaluation, and social skill. Journal of Personality, 63, 315-339.

Peterson, R. A., \& Plehn, K. (1999). Measuring anxiety sensitivity. In S. Taylor (Ed.), Anxiety sensitivity: Theory, research, and treatment of the fear of anxiety (pp. 61-81). Hillsdale, NY: Erlbaum.

Rapee, R. M., \& Heimberg, R. G. (1997). A cognitive-behavioral model of anxiety in social phobia. Behaviour Research and Therapy, 35, 741-756.

Rapee, R. M., \& Lim, L. (1992). Discrepancy between self- and observer ratings of performance in social phobics. Journal of Abnormal Psychology, 101, 728-731.

Reiss, S., Peterson, R. A., Gursky, D. M., \& McNally, R. J. (1986). Anxiety sensitivity, anxiety frequency and the prediction of fearfulness. Behaviour Research and Therapy, 24, 1-8.

Rodebaugh, T. L., \& Chambless, D. L. (2002). The effects of video feedback on self-perception of performance: A replication and extension. Cognitive Therapy and Research, 26, 629-644.

Rodebaugh, T. L., Woods, C. M., Thissen, D. M., Heimberg, R. G., Chambless, D. L., \& Rapee, R. M. (2004). More information from fewer questions: The factor structure and item properties of the original and Brief Fear of Negative Evaluation Scale. Psychological Assessment, 16, 169-181.

Rodriguez, B. F., Bruce, S. E., Pagano, M. E., Spencer, M. A., \& Keller, M. B. (2004). Factor structure and stability of the Anxiety Sensitivity Index in a longitudinal study of anxiety disorder patients. Behaviour Research and Therapy, 42, 79-91.

Saluck, R. G., Herbert, J. D., Rheingold, A., Harwell, V., Coppola, S. W., \& Crittenden, K. (2000, November). The Brief Fear of Negative Evaluation Scale: Preliminary psychometric findings. Poster presented at the annual meeting of the Association for the Advancement of Behavior Therapy, New Orleans, LA.

Smith, R. E., \& Sarason, I. G. (1975). Social anxiety and the evaluation of negative interpersonal feedback. Journal of Consulting and Clinical Psychology, 43, 429.

Spector, P. E., Van Katwyk, P. T., Brannick, M. T., \& Chen, P. Y. (1997). When two factors don't reflect two constructs: How item characteristics can produce artificial factors. Journal of Management, 23, 659-677.

Stanley, M. A., Beck, J. G., \& Zebb, B. J. (1996). Psychometric properties of four anxiety measures in older adults. Behaviour Research and Therapy, 34, 827-838.

Stein, M. B., Jang, K. L., \& Livesley, W. J. (2002). Heritability of social anxiety-related concerns and personality characteristics: A twin study. Journal of Nervous and Mental Disease, 190, 219-224.

Stopa, L., \& Clark, D. M. (1993). Cognitive processes in social phobia. Behaviour Research and Therapy, 31, 255-267.

Stopa, L., \& Clark, D. M. (2001). Social phobia: Comments on the viability and validity of an analogue research strategy and British norms for the Fear of Negative Evaluation questionnaire. Behavioural and Cognitive Psychotherapy, 29, 423-430.

Taylor, S., Woody, S., McLean, P. D., \& Koch, W. J. (1997). Sensitivity of outcome measures for treatments of generalized social phobia. Assessment, 4, 181-191.

Tucker, L. R., \& Lewis, C. (1973). A reliability coefficient for maximum likelihood factor analysis. Psychometrika, 38, 1-10.

Turk, C. L., Heimberg, R. G., \& Hope, D. A. (2001). Social anxiety disorder. In D. H. Barlow (Ed.), Clinical handbook of psychological disorders: A step-bystep treatment manual (3rd ed., pp. 114-153). New York: Guilford Press.

Ventura, J., Liberman, R. P., Green, M. F., Shaner, A., \& Mintz, J. (1998). Training and quality assurance with Structured Clinical Interview for DSM-IV (SCID-I/P). Psychiatry Research, 79, 163-173.

Watson, D., \& Friend, R. (1969). Measurement of social-evaluative anxiety. Journal of Consulting and Clinical Psychology, 33, 448-457.

Wells, A., Clark, D. M., Salkovskis, P., Ludgate, J., Hackmann, A., \& Gelder, M. (1995). Social phobia: The role of in-situation safety behaviors in maintaining anxiety and negative beliefs. Behavior Therapy, 26, 153-161.

Winton, E. C., Clark, D. M., \& Edelmann, R. J. (1995). Social anxiety, fear of negative evaluation and the detection of negative emotion in others. Behaviour Research and Therapy, 33, 193-196.

Zinbarg, R. E., Barlow, D. H., \& Brown, T. A. (1997). The hierarchical structure and general factor saturation of the Anxiety Sensitivity Index: Evidence and implications. Psychological Assessment, 9, 277-284.

Received January 13, 2004 Revision received September 27, 2004 Accepted November 1, 2004 